This item was submitted to Loughborough's Research Repository by the author.

Items in Figshare are protected by copyright, with all rights reserved, unless otherwise indicated.

\title{
Reaction time, cardiorespiratory fitness and mortality in UK Biobank: an observational study
}

PLEASE CITE THE PUBLISHED VERSION

https://doi.org/10.1016/j.intell.2017.11.006

PUBLISHER

(C) Elsevier

VERSION

AM (Accepted Manuscript)

\section{PUBLISHER STATEMENT}

This paper was accepted for publication in the journal Intelligence and the definitive published version is available at https://doi.org/10.1016/j.intell.2017.11.006.

LICENCE

CC BY-NC-ND 4.0

\section{REPOSITORY RECORD}

Yates, Thomas E., Francesco Zaccardi, Nafeesa N. Dhalwani, Kishan Bakrania, Mark Hamer, Kamlesh Khunti, and Melanie J. Davies. 2019. "Reaction Time, Cardiorespiratory Fitness and Mortality in UK Biobank: An Observational Study”. figshare. https://hdl.handle.net/2134/27557. 


\section{Reaction time, cardiorespiratory fitness and mortality in UK Biobank: An observational study}

Total word count main text, references and tables $=3558$

Number of Tables $=1$

Number of Figures $=3$

Supplementary digital content 
Intelligence has previously been associated with mortality, although it is unclear whether associations are independent of related factors such as information processing or cardiorespiratory fitness. We investigate whether fluid intelligence, reaction time and cardiorespiratory fitness are independently associated with mortality within the general population. UK Biobank recruited adults across England, Scotland and Wales, March 2006 to July 2010. 54019 participants (women 52\%) with complete data were included. Those who died in the first year of follow-up $(n=58)$ were excluded. Fluid intelligence was measured as the number of correct answers to a two minute logic/reasoning-test, reaction time was measured as average time taken to respond to matching symbols on a computer screen and cardiorespiratory fitness was measured through a sub-maximal exercise test. Associations with mortality were assessed by Cox-proportional hazard models adjusted for age, sex, ethnicity, social deprivation, cancer and non-cancer illnesses, medications, employment, education, smoking, BMI, diet, sleep, and physical activity. Over 5.8 years of follow-up there were 810 deaths. Higher intelligence (hazard ratio $[\mathrm{HR}]$ per SD $=0.91 ; 95 \% \mathrm{Cl} 0.84,0.99$ ), faster reaction time (HR per SD $=0.92 ; 0.85,0.98)$ ) and higher fitness $(H R$ per $S D=0.85 ; 0.78,0.93)$ were associated with a higher risk of mortality after adjustment for each other and other covariates. No interaction was observed between fluid intelligence and reaction time $(p=0.147)$ or between fluid intelligence and cardiorespiratory fitness $(p=0.238)$. In conclusion, fluid intelligence, reaction time and cardiorespiratory fitness were associated with mortality with estimates persisting after adjustment for each other and multiple risk factors.

Key words: Cardiorespiratory fitness; intelligence, mortality; reaction time 


\section{INTRODUCTION}

Higher levels of intelligence have consistently been associated with a lower risk of mortality across different populations and age groups [1-4]. However, mechanisms supporting these associations have not been well elucidated and confounding or reverse causation remains a possibility. One suggested hypothesis is that higher intelligence might reflect greater 'system integrity' resulting in an organism that can that respond more robustly to environmental stressors [5]. This hypothesise has been explored by several small studies through the use of reaction time, which is as a measure of the brain's information processing efficiency whilst also acting as a wider marker of cognitive function, whole bodily systems integrity and a risk factor for mortality [6-12]. For example, a small epidemiological study found that the association between intelligence and mortality was attenuated after adjusting for reaction time, whilst another study found the association between brain white matter integrity and general intelligence was mediated through measures of reaction time $[6,13]$. Taken together these studies suggest whole body system integrity may support higher order manifestations of cognitive function such as general intelligence whilst acting as the fundamental determinant of health status. However, these studies have not been well replicated within the general population and it remains uncertain whether measures of information processing or whole body system integrity help explain the association between general intelligence and health or whether these exposures are independently associated with health.

Another hypothesise is that along with reflecting greater socioeconomic status, those with higher intelligence are more likely to engage in healthy lifestyle behaviours and thus have better underlying physical health [5]. Cardiorespiratory fitness is one of the most robust measures of physical health status and as such is considered a cardiovascular clinical vital sign $[14,15]$. Importantly cardiorespiratory fitness, physical activity and sedentary behaviour are associated with cognitive function [16-21]. Cardiorespiratory fitness has also been associated with brain function and structure whilst exercise training interventions have been shown to reduce reaction 
time, increase brain volume and improve cognitive functioning $[18,19,22-27]$. Thus it is possible that the association between intelligence and mortality are confounded by cardiorespiratory fitness or vice-versa. Alternatively, cardiorespiratory fitness and intelligence may be correlated through sharing some environmental (i.e. exercise) or genetic determinants but reflect largely different systems of overall health status and therefore act as independent predictors of mortality.

The aim of this paper is to investigate whether intelligence, reaction time and cardiorespiratory fitness are independently associated with all-cause mortality within the general population. .

\section{METHODS}

\section{UK Biobank}

UK Biobank recruited 502,639 individuals living within 25 miles of the 22 study assessment centres located throughout England, Scotland and Wales between March 2006 and July 2010; the aims, methods and assessed outcomes have been reported previously [28]. In brief, UK Biobank is large prospective cohort of middle-aged adults designed to support biomedical research focused on improving the prevention, diagnosis and treatment of chronic disease. Recruited individuals provided comprehensive data on a broad range of biological, demographic, health, lifestyle, mental, social and well-being outcomes. All participants provided written informed consent and the study was approved by the NHS National Research Ethics Service (Ref: 11/NW/0382). This study is part of UK Biobank application number 1081.

\section{Cardiorespiratory fitness}

UK Biobank introduced an ECG monitored sub-maximal exercise test into their suite of physical outcome assessments in each assessment centre towards the end of recruitment. Those with high 
blood pressure (systolic blood pressure $\geq 180 \mathrm{bpm}$ or diastolic blood pressure $\geq 110 \mathrm{bpm}$ ), chest pain, or pregnant women were excluded from the test. The test was terminated if $75 \%$ of age-predicted maximum heart rate was reached. The test involved 2 minutes at a constant workload followed by 4 minutes of a linearly increasing workload. The start and end workload of the graded exercise test were standardised according to age, height, weight, resting heart rate and sex to ensure a similar relative intensity across the population. Heart rate was monitored before, during, and after the exercise test via a 4-lead ECG. Maximal cardiorespiratory fitness was estimated using previous published criteria [29], involving: 1) Fitting a linear regression line between heart rate and power output for each stage of the test; 2) Extrapolating the regression line to the age-predicted maximal heart rate with the formula: $208-0.7 \times$ age; 3) Using the regression equation for the relationship between work rate (power) and oxygen uptake (oxygen uptake [ml.kg-1.min-1] $=7+(10.8 \times$ work rate $[$ Watts])/body mass $[\mathrm{kg}]$ ) to estimate maximal oxygen uptake. Metabolic equivalents (METs) were calculated through dividing maximal oxygen update by 3.5 .

\section{Fluid Intelligence}

Fluid intelligence was assessed through 13 questions using verbal and numeric reasoning/logic delivered over a two minute period. Each question had five possible responses in addition to "do not know" and "prefer not to answer"; participants were asked to select the correct response via a touch screen computer. Participants were instructed as follows: "In this next test you will have a maximum of two minutes to answer as many questions as possible. Don't spend too long on any one question and you can skip any question if you wish". Each question used in the test is available through the UK Biobank website [30]. The number of correct responses was used as the outcome measure. This measure of fluid intelligence employed in UK Biobank has been shown to have good reliability when repeated after a mean of 4 years $($ ICC $=0.65 ; p<0.001)[31]$. 


\section{Reaction time}

Simple reaction time was assessed with a timed test of symbol matching conducted on a touch screen computer, similar to the card game snap. Two card shapes with symbols were displayed on a computer screen with participants asked to press a red button with their dominant hand if the two cards had matching symbols. Participants undertook the test seated with the red button placed on a desk in front of them. Ten symbols with simple non-complex shapes were used (equals sign, fir tree, hollow circle, hollow square, $\mathrm{H}$, smiley face, solid circle, solid square, triangle, cross). Participants completed 12 rounds, of which rounds the first 5 rounds were considered training rounds and removed from the analysis. Of the remaining rounds, matched cards were shown in rounds $6,8,11$ and 12 . Reaction time was calculated as the average time taken to correctly identify a match. Times under $50 \mathrm{~ms}$ were considered to be due to anticipation rather than reaction and removed from the analysis. Participants were allowed 2000 ms to react to each round.

\section{Anthropometric, demographic, health and lifestyle data}

This study utilised the following covariate data within UK Biobank: anthropometric (body mass index (BMI)); demographic (age, sex, ethnicity, social deprivation [Townsend index], employment status and education level); health status (prevalent cancer, number of prevalent non-cancer illnesses, and number of prescribed medications); and lifestyle (smoking [never, past, current], alcohol, fresh fruit [pieces per day], raw vegetables or salad [portion per day], cooked vegetable [potion per day], sleep [hours per night], TV viewing time [hours per day], leisure computer use [hours per day], and physical activity [weekly frequency of any walking, moderate-, or vigorous-intensity physical activity undertaken lasting at least 10 minutes]. Alcohol was assessed through a frequency questionnaire ranging from never to daily or almost daily. Further details for each measure are available elsewhere[28]. 


\section{Assessment of mortality status and data inclusion}

UK Biobank undertook comprehensive data linkage for mortality status using national records in England, Wales and Scotland. Using a unique identifier collected at baseline (e.g. NHS number), information about mortality status, date of death and causes of death were obtained from National Health Service (NHS) Information Centre for participants from England and Wales, and from the NHS Central Register, for participants from Scotland. Linkage captured all deaths occurring until $31^{\text {st }}$ January 2016 for England and 30

\section{Data inclusion}

This study only includes those that were assigned to and completed at least one stage of the exercise test $(n=59068)$. Those who died within the first 12 months following their visit to the assessment centre $(n=58)$ were removed from the analysis to reduce the possible impact of reverse causation. Given the presence of missing covariate data were low (less than $5 \%$ for all included covariates), analysis were further restricted to those with complete covariate data (Supplementary Digital Content 1).

\section{Statistical analysis}

Descriptive variables were tabulated and reported as median (interquartile range, IQR) or number (\%). Spearman rank correlation was used to test the strength of correlation between fluid intelligence, reaction time and cardiorespiratory fitness. Intra-class correlation coefficients were used to assess the reliability of reaction time across each round compared to the reported average value. Cox-proportional hazard models were used to investigate the association of fluid intelligence, reaction time, and cardiorespiratory fitness and with mortality. As neither reaction time nor cardiorespiratory fitness were normally distributed, natural logarithm transformed values were used (Supplementary Digital Content 2). In order to allow the comparison of associations for fluid intelligence, reaction time and fitness, results are reported per 1-SD (standard deviation). Data for reaction time was inverted to show the same direction as cardiorespiratory fitness and fluid 
intelligence and correspond to per 1-SD lower (faster) values. For descriptive purposes, the value for the SD for cardiorespiratory fitness and reaction time is reported as the difference between the geometric mean and the geometric mean plus one SD. Progressive adjustment for covariates were undertaken: model 1 adjusted for age, sex and ethnicity; model 2 further included demographic factors as social deprivation, prevalent cancer, number of non-cancer illness, number of medications, employment status, and education level. Model 3 additionally adjusted for the following lifestyle factors: smoking status, BMI, fruit intake, cooked vegetable intake, salad and raw vegetable intake, alcohol intake, sleep time, walking activity, moderate-intensity physical activity, vigorous-intensity physical activity. Finally, model 4 was further mutually adjusted for both cardiorespiratory fitness and reaction time to investigate whether the association of one with mortality was independent of the other. Interaction terms were fitted to model 4 to test whether the association between reaction time and mortality was modified by cardiorespiratory fitness and vice-versa. For descriptive purposes and in order to display the associations, categories of fluid intelligence (low, average, high), cardiorespiratory fitness (low, average, high) and reaction time (slow, average, fast) were created using sex-specific tertiles. Cox-proportional hazard models were used to investigate the pattern of association across increasing categories of fluid intelligence and cardiorespiratory fitness and decreasing categories of reaction time. Analysis were performed with SPSS (version 24) and results reported with $95 \%$ Cis unless stated otherwise.

\section{Sensitivity analysis}

Analysis for the fully adjusted model (model 4) was repeated after removing those with prevalent cancer, cardiovascular disease (angina, myocardial infarction, and/or stroke) or cognitive/psychiatric disease (neurological injury/trauma, psychological/psychiatric condition, chronic/degenerative neurological condition, motor neurone disease, multiple sclerosis, Parkinson's disease, dementia/Alzheimer's disease/cognitive impairment, depression, anxiety/panic attacks, nervous 
breakdown, schizophrenia, deliberate self-harm/suicide attempt, and/or mania/bipolar disorder/manic depression).

\section{RESULTS}

This study reports data from 54019 participants with complete cardiorespiratory fitness, reaction time and covariate data (Supplementary Digital Content 1). Supplementary Digital Content 3 displays characteristics of included participants compared to the full UK Biobank dataset. For included participants, the median (IQR) for fluid intelligence, reaction time and cardiorespiratory fitness were $6(5,8)$ correct answers, $538(480,613)$ milliseconds and $9.9(8.2,12.0)$ METs respectively. Corresponding values for age and BMI were $58(50,63)$ years and $26.5(24.0,29.5) \mathrm{kg} / \mathrm{m}^{2}$ respectively, whilst $52 \%$ were women and the majority (85\%) were White European. The distribution of reaction time, age, BMI, sex and ethnicity were similar to the full UK Biobank cohort (Supplementary Digital Content 3).

Fluid intelligence, reaction time and cardiorespiratory fitness were weakly correlated ( $\rho$ between 0.12 and 0.17 for all combinations), see Table 1 . Average reaction time showed good reliability (ICC $=0.82 ; 0.82,0.83)$.

During a median follow-up of $5.8(5.6,5.8)$ years, 779 incident deaths occurred. Fluid intelligence, reaction time and cardiorespiratory fitness were all associated with all-cause mortality (Figure 1). After adjustment for reaction time and cardiorespiratory fitness along with demographic, medical and lifestyle factors, the HR for the association between higher fluid intelligence and mortality was $0.91(0.84,0.99)$ per SD (Figure 1). The corresponding values per SD faster reaction time and per SD higher cardiorespiratory fitness were $0.92(0.85,0.98)$ and $0.85(0.78,0.93)$ respectively (Figure 1$)$. For the average individual within the population, per SD equated to a difference of 3.1 METs for cardiorespiratory fitness, $\mathbf{1 1 0} \mathrm{ms}$ for reaction time and $\mathbf{2}$ additional correct answers for fluid 
intelligence. No interaction was observed between fluid intelligence and reaction time $(p=0.147)$ or between fluid intelligence and cardiorespiratory fitness $(p=0.238)$.

For descriptive purposes, Supplementary Digital Content 4 shows the association of fluid intelligence, reaction time and cardiorespiratory fitness with mortality when categorised into tertiles. Those in the highest tertile of fluid intelligence had a $20 \%(2 \%, 34 \%)$ lower risk of mortality compared to those in the lowest tertile. Similarly those in the fastest tertile of reaction time and those in the highest tertile of cardiorespiratory fitness had a $19 \%(2 \%, 33 \%)$ and $73 \%(11 \%, 40 \%)$ compared to those in the lowest tertiles.

Sensitivity analysis

The results were not affected if those with prevalent cancer, cardiovascular disease or cognitive/psychiatric disease $(\mathbf{n}=\mathbf{9 5 3 1} \mathbf{1 8 \%})$ were removed from the analysis (Supplementary Digital Content 5).

\section{DISCUSSION}

This study found that fluid intelligence, reaction time and cardiorespiratory fitness were independently associated with all-cause mortality. Specifically, the association between fluid intelligence and mortality was robust against adjustment for lifestyle behaviours and sociodemographic factors and remained unaffected by further adjustment for reaction time and cardiorespiratory fitness. In the fully adjusted model, each SD higher fluid intelligence score was associated with a $9 \%$ reduction in mortality risk. Similarly, each SD faster reaction time and each SD higher cardiorespiratory fitness levels were associated with an $8 \%$ and $15 \%$ reduction in the risk of all-cause mortality respectively.

This study extends previous research through showing that the association between intelligence and mortality is independent of both reaction time (as a marker of information processing efficacy and whole body system integrity) and cardiorespiratory fitness (a measure of physical health). Our 
findings are in contrast to another study which reported that the association between intelligence and mortality was attenuated after adjustment for reaction time [6]. However, this previous study had a small sample size $(n=898)$ and is likely to have been under powered for this analysis. For example although non-significant, each SD lower intelligence score was still associated with a $20 \%$ higher risk of mortality after adjustment for reaction time [6]. Our study in a large well characterised cohort suggests that whilst reaction time and intelligence were correlated, adjustment for reaction time had a negligible impact on the association between intelligence and mortality. Further, reaction time was itself independently associated with mortality after adjustment for intelligence. Therefore intelligence and reaction time may work through different pathways in the association with mortality.

A further important finding from this study was that the associations of fluid intelligence and reaction time with mortality was not attenuated after adjustment for cardiorespiratory fitness. Cardiorespiratory fitness is a well-researched and established measure of overall physical health status, with this study supporting previous meta-analyses reporting that each additional 1 MET in cardiorespiratory fitness level is associated with a $13 \%$ lower risk of all-cause mortality [15], a $15 \%$ lower risk of cardiovascular disease [15], and a 5\% lower risk of type 2 diabetes [32]. It has previously been suggested that differences in health behaviour or health status may confound or explain part of the association of intelligence with mortality [5]. Our findings suggest that overall physical health status may in fact have little impact on these associations and that therefore intelligence represents an independent predictor of mortality risk. These findings are consistent with previous research that have shown that the association of both intelligence and reaction time with mortality are independent of other measures of physical health, such as blood pressure or factors relate to the metabolic syndrome[7,33].

The present study has important strengths, including the first study to include the joint analysis of intelligence, reaction time and cardiorespiratory fitness. However, limitations remain. Firstly, 
although we removed deaths occurring in the first 12 months and undertook a sensitivity analysis also excluding those with related chronic disease, reverse causality remains a possibility. Reaction time may only be a crude measure of information processing or bodily system integrity; however it is a commonly used out in psychometric research. Fitness was measured through a sub-maximal rather than maximal test and thus may have increased measurement error and increased the risk of regression dilution. However, submaximal measures of cardiorespiratory fitness have been shown to correlate reasonably with maximal tests across different populations and are widely used in epidemiological research [34]. The fitness test was introduced towards the end of UK Biobank recruitment and was therefore only available on a sub-set of participants. In addition, those with high cardiovascular disease risk, such as significantly elevated blood pressure, were excluded from the test, potentially resulting in a healthier than average population which may in turn have led to more conservative estimates. Nevertheless, the distribution of age, sex, ethnicity and reaction time in the cohort included in this study was similar to the full UK Biobank cohort. Finally, although we adjusted for a wide range of demographic, anthropometric, clinical and lifestyle factors, residual confounding could still be present.

In conclusion, this study highlights that fluid intelligence and simple reaction time are predictors of all-cause mortality which are independent of each other and cardiorespiratory fitness. Future clinical trials should consider the inclusion of simple psychometric measures, such as those used in UK Biobank, to help further elucidate the importance of these factors in determining health status.

\section{ACKNOWLEDGEMENTS}

Other Conflicts of Interest: None

Reviewers: We thank the Reviewers for this paper, who contributed significantly to the inclusion and interpretation of data. 
Data source: This research has been conducted using the UK Biobank Resource under Application Number 1081 


\section{References}

1. Calvin CM, Deary IJ, Fenton C, et al. Intelligence in youth and all-cause-mortality: Systematic review with meta-analysis. Int J Epidemiol. 2010;40(3):626-644.

2. Christensen GT, Mortensen EL, Christensen K, Osler M. Intelligence in young adulthood and causespecific mortality in the danish conscription database-a cohort study of 728,160 men. Intelligence. 2016;59:64-71.

3. Iveson MH, Čukić I, Der G, Batty GD, Deary IJ. Intelligence and all-cause mortality in the 6-day sample of the scottish mental survey 1947 and their siblings: Testing the contribution of family background. Int J Epidemiol. 2017:dyx168.

4. Batterham PJ, Christensen H, Mackinnon AJ. Fluid intelligence is independently associated with allcause mortality over 17 years in an elderly community sample: An investigation of potential mechanisms. Intelligence. 2009;37(6):551-560.

5. Deary I. Why do intelligent people live longer? Nature. 2008;456(7219):175-176.

6. Deary IJ, Der G. Reaction time explains IQ's association with death. Psychol Sci. 2005;16(1):64-69.

7. Roberts BA, Der G, Deary IJ, Batty GD. Reaction time and established risk factors for total and cardiovascular disease mortality: Comparison of effect estimates in the follow-up of a large, UK-wide, general-population based survey. Intelligence. 2009;37(6):561-566.

8. Hagger-Johnson G, Deary IJ, Davies CA, Weiss A, Batty GD. Reaction time and mortality from the major causes of death: The NHANES-III study. PloS one. 2014;9(1):e82959.

9. Deary IJ. Looking for 'system integrity' in cognitive epidemiology. Gerontology. 2012;58(6):545553. 
10. Nissan J, Liewald D, Deary IJ. Reaction time and intelligence: Comparing associations based on two response modes. Intelligence. 2013;41(5):622-630.

11. Shipley BA, Der G, Taylor MD, Deary IJ. Cognition and all-cause mortality across the entire adult age range: Health and lifestyle survey. Psychosom Med. 2006;68(1):17-24.

12. Batterham PJ, Bunce D, Mackinnon AJ, Christensen $\mathrm{H}$. Intra-individual reaction time variability and all-cause mortality over 17 years: A community-based cohort study. Age Ageing. 2014;43(1):8490.

13. Penke L, Maniega SM, Bastin M, et al. Brain white matter tract integrity as a neural foundation for general intelligence. Mol Psychiatry. 2012;17(10):1026-1030.

14. Ross R, Blair SN, Arena R, et al. Importance of assessing cardiorespiratory fitness in clinical practice: A case for fitness as a clinical vital sign: A scientific statement from the american heart association. Circulation. 2016:CIR. 0000000000000461.

15. Kodama S, Saito K, Tanaka S, et al. Cardiorespiratory fitness as a quantitative predictor of allcause mortality and cardiovascular events in healthy men and women: A meta-analysis. JAMA. 2009;301(19):2024-2035.

16. Hillman $\mathrm{CH}$, Erickson $\mathrm{KI}$, Kramer AF. Be smart, exercise your heart: Exercise effects on brain and cognition. Nature reviews neuroscience. 2008;9(1):58-65.

17. McAuley E, Szabo AN, Mailey EL, et al. Non-exercise estimated cardiorespiratory fitness: Associations with brain structure, cognition, and memory complaints in older adults. Mental health and physical activity. 2011;4(1):5-11.

18. Barnes DE, Yaffe K, Satariano WA, Tager IB. A longitudinal study of cardiorespiratory fitness and cognitive function in healthy older adults. J Am Geriatr Soc. 2003;51(4):459-465. 
19. Freudenberger $P$, Petrovic $K$, Sen $A$, et al. Fitness and cognition in the elderly: The austrian stroke prevention study. Neurology. 2016;86(5):418-424.

20. Bakrania K, Edwardson CL, Khunti K, Bandelow S, Davies MJ, Yates T. Associations between sedentary behaviours and cognitive function: Cross-sectional and prospective findings from the UK biobank. Am J Epidemiol. 2017.

21. Sabia S, Guéguen A, Marmot MG, Shipley MJ, Ankri J, Singh-Manoux A. Does cognition predict mortality in midlife? results from the whitehall II cohort study. Neurobiol Aging. 2010;31(4):688-695.

22. Colcombe SJ, Erickson KI, Scalf PE, et al. Aerobic exercise training increases brain volume in aging humans. J Gerontol A Biol Sci Med Sci. 2006;61(11):1166-1170.

23. Colcombe SJ, Kramer AF, Erickson KI, et al. Cardiovascular fitness, cortical plasticity, and aging. Proc Natl Acad Sci U S A. 2004;101(9):3316-3321.

24. Erickson KI, Voss MW, Prakash RS, et al. Exercise training increases size of hippocampus and improves memory. Proc Natl Acad Sci U S A. 2011;108(7):3017-3022.

25. Ponce-Bravo H, Ponce C, Feriche B, Padial P. Influence of two different exercise programs on physical fitness and cognitive performance in active older adults: Functional resistance-band exercises vs. recreational oriented exercises. J Sports Sci Med. 2015;14(4):716-722.

26. Predovan D, Fraser SA, Renaud M, Bherer $L$. The effect of three months of aerobic training on stroop performance in older adults. J Aging Res. 2012;2012:269815.

27. Rooks DS, Kiel DP, Parsons C, Hayes WC. Self-paced resistance training and walking exercise in community-dwelling older adults: Effects on neuromotor performance. J Gerontol A Biol Sci Med Sci. 1997;52(3):M161-8. 
28. UK Biobank. UK biobank: Protocol for a large-scale prospective epidemiological resource.

http://www.ukbiobank.ac.uk/wp-content/uploads/2011/11/UK-Biobank-Protocol.pdf. Updated 2007. Accessed 01/12, 2017.

29. Celis-Morales CA, Lyall DM, Anderson J, et al. The association between physical activity and risk of mortality is modulated by grip strength and cardiorespiratory fitness: Evidence from 498135 UKbiobank participants. Eur Heart J. 2017;38(2):116-122.

30. UK Biobank. UK biobank website. https://www.ukbiobank.ac.uk/. Updated 2017. Accessed 01/20, 2017.

31. Lyall DM, Cullen B, Allerhand M, et al. Cognitive test scores in UK biobank: Data reduction in 480,416 participants and longitudinal stability in 20,346 participants. PloS one. 2016;11(4):e0154222.

32. Zaccardi F, O'Donovan G, Webb DR, et al. Cardiorespiratory fitness and risk of type 2 diabetes mellitus: A 23-year cohort study and a meta-analysis of prospective studies. Atherosclerosis. 2015;243(1):131-137.

33. Batty G, Gale C, Mortensen LH, Langenberg C, Shipley M, Deary I. Pre-morbid intelligence, the metabolic syndrome and mortality: The vietnam experience study. Diabetologia. 2008;51(3):436-443.

34. Sartor F, Vernillo G, De Morree HM, et al. Estimation of maximal oxygen uptake via submaximal exercise testing in sports, clinical, and home settings. Sports medicine. 2013;43(9):865-873.

35. Lin X, Zhang X, Guo J, et al. Effects of exercise training on cardiorespiratory fitness and biomarkers of cardiometabolic health: A systematic review and meta-analysis of randomized controlled trials. J Am Heart Assoc. 2015;4(7):10.1161/JAHA.115.002014.

36. Kodama S, Tanaka S, Saito K, et al. Effect of aerobic exercise training on serum levels of highdensity lipoprotein cholesterol: A meta-analysis. Arch Intern Med. 2007;167(10):999-1008. 
37. Cornelissen VA, Smart NA. Exercise training for blood pressure: A systematic review and metaanalysis. J Am Heart Assoc. 2013;2(1):e004473.

38. Ahmed HM, Blaha MJ, Nasir K, Rivera JJ, Blumenthal RS. Effects of physical activity on cardiovascular disease. Am J Cardiol. 2012;109(2):288-295. 
Table 1: Spearman's correlation coefficient matrix between intelligence, reaction time and cardiorespiratory fitness

\begin{tabular}{|llll|}
\hline & Intelligence & Reaction time & Cardiorespiratory fitness \\
Intelligence & - & $-0.16^{*}$ & $0.12^{*}$ \\
Reaction time & $-0.16^{*}$ & - & $-0.17^{*}$ \\
Cardiorespiratory fitness & $0.12^{*}$ & $-0.17^{*}$ & - \\
\hline
\end{tabular}

$* p<0.001$ 
Figure 1: Associations of fluid intelligence, reaction time and cardiorespiratory fitness with all-cause mortality

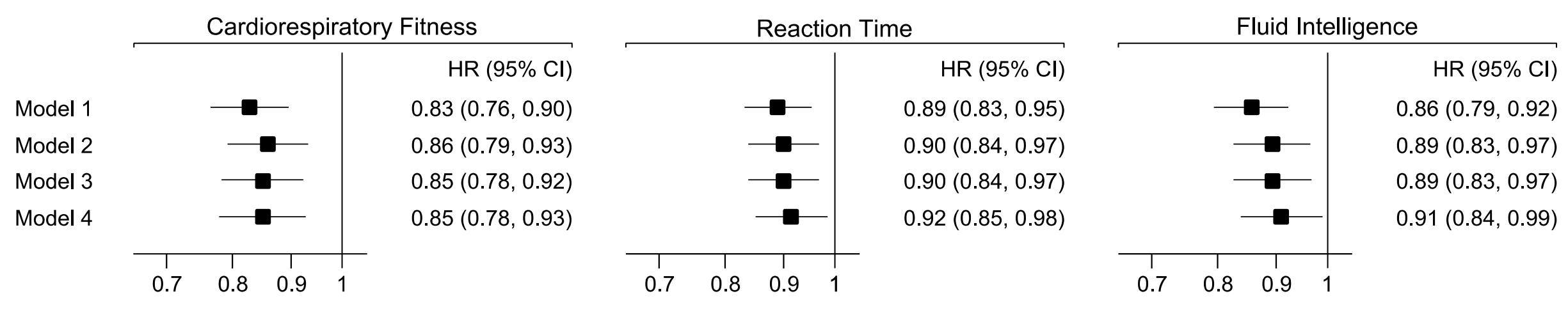

Data show HR per SD high fluid intelligence, fasting reaction time and higher fitness

Model 1: Adjusted for age, sex and ethnicity,

Model 2: Adjusted for age, sex, ethnicity, social deprivation, prevalent cancer, number of non-cancer illness, number of medications, employment status, education level

Model 3: Adjusted for age, sex, ethnicity, social deprivation, prevalent cancer, number of non-cancer illness, number of medications, employment status, education level, smoking status, BMI, fruit intake, cooked vegetable intake, salad and raw vegetable intake, alcohol intake, sleep, TV viewing, walking activity moderate-intensity activity, vigorous-intensity activity

Model 4: Adjusted for Model 3 plus mutually adjusting for fluid intelligence, reaction time and cardiorespiratory fitness 